\title{
New literacies and future educational culture
}

\author{
Anita Pincas \\ Institute of Education, University of London \\ email:a.pincas@ioe.ac.uk
}

The paper draws attention to three developments that are crucial to online education. First, the new literacy required by group discussion in writing, i.e by computer-mediated communication ('e-talk') is discussed. Educators are urged to delimit and structure their courses so that online conversations in writing are successfully framed for effective discourse. Second, new literacy arising from the merging of multimedia with text is considered. It is maintained that this will enhance communication, not debase it. Third, the way that increasing ease of information retrieval is eroding boundaries between traditional disciplines is discussed. It is argued that this may create new difficulties in education. The paper recommends various ways of overcoming the problems that arise from the three developments.

\section{Introduction}

The argument in this paper is based on the view that, even though traditional classroom teaching and lecturing are likely to remain the norm, there is a recognized and growing place for the use of technology in educational provision in schools and universities. Online course design is still very much open to discussion, since traditions of delivery have yet to develop. But new software developments are arriving so quickly, that educators have barely time to experiment with one mode of delivery before they are overtaken by another.

There are three issues that are, or shortly will be, crucial to online education:

- the new literacy required by group discussion in writing - 'e-talk', and developments in voice-recognition technology as well as automatic translation;

- a second new literacy arising from the merging of multimedia with text, heralding new hybrid artefacts of communication, which, moreover, the reader has the power to alter; 
- the increasing ease of information retrieval, mainly keyword searches of the Web and citation indices, both of which are steadily contributing to the erosion of boundaries not only between traditional disciplines, but between published and informal discourse.

One issue that must urgently be addressed is how to determine our priorities in order to help educators, as well as the disenfranchised, the unemployed and the alienated. Should we make efforts to improve the cheapest, simplest and until now most reliable of text-based international communications, namely email, or should we rather turn our attention to the potentials of the most exciting new multimedia on the Web with its attendant new literacy demands.

Although the above three developments are at first sight apparently unrelated, they are very likely to blend and thus evolve further new paradigms of literacy, knowledge and education. The problem has of course been recognized in outline for some time, for instance by Meyrowitz (1985), who described how the new kinds of place created by television and computers led to a separation of social from physical place, a blurring both of public and private behaviours and of adulthood and childhood, the merging of genders, and the appearance of new group identities. Although he acknowledged that there were limitations as well as new freedoms in the electronic age, he maintained that, on the whole, to be liberated from the traditions of space-determined encounters and print-controlled information was a healthy development. He foresaw how traditional expertise based on the 'one-thing-at-a-time, one-thing-after-another and take-time-to-think' world of reading (p. 326) might give way to more valuable types of thinking which involve both the integration of multiple variables and overlapping lines of simultaneous actions' as in video-games (p. 326) and the breaking of discipline boundaries because of universal equal access to data from all fields via television and computer searches (p. 327).

Today, such changes are even more clearly foreseeable. But an endorsement of the value of technology does not imply an uncritical acceptance of it. Recent arguments (Noble 1997, Hammonds, Jackson, George, and Morris, 1997) have highlighted the perhaps unfortunate role of business-driven initiatives in some US universities. It is crucial for us to clarify our own educational priorities, especially by looking ahead. In this context each of the three new developments will be discussed in turn and some possible effects on the culture of education predicted. The interrelationship between all three will be suggested.

\section{E-talk}

Over the last few years, email has become established in the academic and the general community, while Web-based communication systems are moving towards a seamless interface between Web information and interaction among people. Online discussion is a new genre, which I have elsewhere called 'e-talk' - electronic conversations in writing (Pincas, 1999). It has features different from both spoken and written language (Yates, $1993 ; 1995)$ and it presents users with unaccustomed problems.

The rapid emergence of voice-text systems (Hershman, 1997) will to some extent change the situation again, first, by bypassing the keyboard, and second, by shifting the user's language from the written mode to the spoken, even though the receiver might still elect to read a text version rather than listen to speech. Both of these factors will contribute to the faster development of educational delivery by computer-mediated communication (CMC). 
For the moment, however, problems of conversation in writing are paramount. They spring from the apparent formless nature of CMC discussions, as perceived by inexperienced users, and also from the stark absence of expected paralinguistic effects. This section will propose ways of lessening the difficulties, and will deal briefly with crosscultural effects on communication.

It is necessary to recognize the division between two ways of using the Internet (which can still be divided roughly into email and the Web), namely open or closed. Open email is exemplified by chat lines (informal synchronous computer-mediated conversations in writing), MOOs and MUDs (slightly more structured synchronous computer-mediated conversations in writing, with attempts to create imaginary, virtual rooms and other spaces), and list-server discussions that anyone can join. In any of these environments, people do not normally know each other in any concrete reality but nevertheless communicate about a range of topics including their own lives and emotions. Closed email is exemplified by private communications and online discussions deliberately initiated for a fixed, specified and controlled group of people who may or may not know each other in the 'real' face-to-face world, but who at least can locate each other by the co-ordinates of that 'real' world, i.e. a name, an occupation, a phone number, a partial address, etc.

The distinction between open and closed email is not absolute, since there are varying degrees of each, e.g. a chatline, MOO or list-server designed for members of a certain profession or followers of a certain hobby, is partly closed, whereas a discussion conference for a group of students on a course may admit non-registered students as guests who are not known to the student group. The Web may likewise be open when Web pages offer unrestricted access to anyone, but are closed in differing degrees as registration or routing through a moderator are required.

\section{Structuring online discussion}

The open vs. closed contrast is crucial for any educational initiative that attempts to function partly or wholly online. Open contexts are essentially unstructured and uncontrolled. It is well attested in CMC literature (Archee, 1993; Coombes, 1993; Shedletsky, 1993) that insufficiently structured open-ended discussions online are more difficult than in ordinary speech contexts where time limitations restrict participation and instant feedback can limit digressions. Online conversations are characterized by multiple threads of content, proceeding fugue-like over elongated time periods, and by the ability of all members to take part without being interrupted. Early studies of CMC in education frequently reported on these features, calling them overload or, more recently, 'infoglut " (Harasim, Hiltz, Teles and Turoff, 1997, p. 222). In a context that values student-studenttutor dialogue as an element of the learning process, where, therefore, language is central to collaborative learning (Laurillard, 1993), an open situation can indeed lead to overwhelming chaos.

To overcome this difficulty, an online teacher has to take effective steps to close and frame the education in general and online conversations in particular. One way of doing this is to fragment topics so that discussion will remain relevant. Another way is to regulate online conversations, making sure that students know very precisely what is required of them. Yet another solution is development of appropriate discourse conventions for group communication between remote interlocutors. Whether assisted by voice-text mechanisms 
or not, the conventions that will suit the new context need to be different from those of ordinary conversation. In the present culture of education where courses are being marketed globally, strangers learning together in CMC courses are breaking into new uses of language and forging new norms of interaction.

However, the best main advice present is to close and structure any online discussion. After all, ordinary conversation is manageable because of learned conventions, principally those of turn-taking. These conventions are severely altered by what is possible in CMC. The advice that follows will briefly outline communication problems in CMC, relying on a classification provided by Poyatos (1983) of turn-taking behaviours when interlocutors are either facing each other or hearing each others' voices (henceforth referred to as FV). A 'turn' is an occasion for speaking.

The discussion in this section assumes currently available $\mathrm{CMC}$ technology. Looking further ahead, text with linked or incorporated visual and sound elements may overcome some of the difficulties discussed and we may see the birth of yet another kind of discourse where CMC conversation will share features of real-life encounters. As in early, unconsidered, uses of CMC, we will at first see an open use of them. Later, it will be understood that conventions of framing and regulation need to be imposed, otherwise the chaos of free-for-all activity will have its negative effect.

\section{Initial turns}

An initial turn in FV may be claimed by physical movement, e.g. pulling up a chair, standing up, taking another's arm, throat clearing, eye contact etc., or by a verbal move such as saying 'Well . . .' It may be granted by a listener as an offering, e.g. when a listener indicates willingness by smiling, pointing, leaning back, or using words such as 'Go ahead'.

CMC involves a quite radical contrast: anyone is able to send a message at any time, but norms of behaviour can be established within a certain group, just as they are in many FV contexts such as committee meetings. For example, the tutor could nominate someone to start a discussion. However, even if such a proposal is made, any other student could none the less take the first turn, saying, for instance, 'Before John Doe starts the ball rolling, could I just ask . . $\therefore$, and might thereby pre-empt totally what the tutor had wanted. A tutor or moderator in CMC might have the power to delete another's contribution, but could probably not prevent some members of the group from having already seen it.

Many topics may be initiated by different members of the group, and the result can very quickly become a complex web of criss-crossing initiations and responses which even the participants find difficult to negotiate, and may very soon abandon. The lesson for moderators of $\mathrm{CMC}$ educational interactions is to be aware of the consequences of fostering open discussions without pre-defined turn-taking roles.

\section{Turn changes}

Turn changes in FV are initiated by the listener, as a bid to take the floor, and may be very similar to initial turns. The speaker has then the option of yielding the floor. The listener may have preceded his attempt to take the floor by pre-opening moves such as signals such as 'Er ...' or physical movement, etc. 
In CMC, it is not a question of listener-bid and speaker-yielding, but, rather, it is at least theoretically possible that every member of the group in a free-flowing discussion will respond, and then that every member will respond to every response! In practice, of course, one might make an informed guess that one-fifth of the group will respond each time, because the asynchronicity guarantees that at least some readers will see early responses to the opening turn and will not feel any need to add to the discussion themselves. None the less, even a small progression of successive responses can quickly become a very large number of messages. In addition, the CMC listener has an opportunity to respond more fully than the FV listener and may therefore steer the discussion into a new direction, thus taking over from the speaker, who may then be at pains to bring it back to the line he started.

In other words, any CMC response could function as the opening of a new thread of discussion, since no one can 'hold' the floor. Whereas there is a variety of further FV turnchange behaviours, e.g. speaker nomination of the next speaker, or speaker overruling a listener's attempt to take the floor, the standard case in CMC is that the speaker always loses the floor once the message has been sent. It is indeed questionable to what extent there is indeed a floor to be held in CMC. It would have to be established by some kind of, probably prior, common agreement. For instance, students might know that they should always defer to the instructor. Or, again, they might feel that one of their own number has higher status or greater knowledge, and therefore should be shown deference. Such deference might be signalled variously, e.g. in relative brevity of responses to the speaker's message(s), or in overt encouragement to the speaker to continue ('I would like to hear your views on ...'), or in covert admission of the speaker's dominance ('I found your message ... very interesting John. Thank you').

More importantly, because of the prevalence of multiple strands of discussion, the floor in $\mathrm{CMC}$ has to be defined in terms of threads of messages and their content. A sequence of messages can be called a thread if there is evidence that the senders intend them to relate to each other and they do relate in content. Current software and user inexperience still make this problematic, however. A listener may reply to a speaker using the software's reply function, indicating his or her intention to continue a thread. However many respondents carelessly use the reply function merely to communicate with the sender about something else, often without bothering to change the subject header.

None the less, even in closed CMC discussion, there is normally a number of floors, or threads. In other words the unique contribution of CMC to educational discourse is the opportunity for a group of participants to run several sub-discussions at the same 'time', in each of which there might be different speakers holding the floor and different patterns of turn-taking. As has been indicated earlier, the larger the volume of such discussions, the more difficult it becomes to follow, even for those most intimately involved in it, again arguing strongly for a good structure within which overload is less likely to occur.

\section{Sequences of turns}

It is evident that sequences of turns have a unique character in CMC. There are adjacency pairs as in FV ('I think X' - 'So do I', or 'Will you come?' - 'Yes, OK') but they may not appear next to each other in the CMC list of messages. It has been suggested that CMC users compensate for the lack of FV discourse management strategies by an increased use 
of openers in order to achieve interaction, e.g. questions, suggestions, requests and an increased use of expressions like 'I'm sending you this message because . . .', 'You can decide ...', 'What do you think?' ( Condon and Cech, 1996).

\section{Listener feedback}

A particular problem in CMC is listener behaviour generally. In FV, listener feedback is likely to involve a whole family of behaviours, e.g. attention signals, such as silence, verbal items like 'Oh' or 'Yes', kinesic indication such as leaning forward, adjusting the facial expression, etc. But CMC users often wonder if anyone is listening to them (i.e. reading their messages). They often follow a message with another one asking 'Have you read my message of ...?' or 'Does anyone agree or disagree with what I wrote on ...?' or even 'No one has responded to my message of ... Is there something wrong?' In time, they develop group behaviours to overcome this, but only within a properly framed discourse structure.

In FV there is also considerable interlistener behaviour, with or without the speaker noticing, to express their reactions, or compete or prompt each other to take the next turn. This in principle is possible in CMC, e.g. by private email, but less effective since it takes very much longer than in FV.

Fortunately, language users are flexible and creative and therefore adapt their practices to the needs of new contexts, whether CMC or any other social context. Educators should help the process by defining roles, making conventions explicit, and generally raising awareness. Even such an apparently minor matter as whether it is acceptable to simplify typed text by abbreviations of words and short syntactic constructions needs to be made clear (Condon and Cech, 1996).

\section{Cultural aspects of online discussion}

The discussion has assumed that CMC writers were English speakers. But a totally different kind of CMC literacy problem is developing as universities add more overseas students to their lists; they are discovering cross-cultural issues that are compounded in $\mathrm{CMC}$ because English is either a foreign language or a second language in use in countries like India, Singapore or Kenya, where local uses have led to new versions of it (Kachru, 1983). Overseas students will have their own attitudes to ways of using different genres with different topics, different notions of what is or is not appropriate to say and in what form of speech, and even different uses of silence.

It is a partial benefit that some cultural differences are not very apparent online. Accents, behaviour, race, religion and the other elements that make up cultural diversity are filtered out by the graphic text and have a levelling effect on status (Davis and Brewer, 1997). On the other hand, users may be quite unaware of misunderstandings and problems that individuals face, unless they are overtly mentioned. Again, explicit consciousness-raising could be a solution.

For the English-speaking world the norms of discourse have been encapsulated by one of the most cited writers within linguistics (Grice, 1975), who put forward the maxims of the Co-operative Principle:

- Make your contribution as informative as required; do not make your contribution more informative than required. 
- Make your contribution relevant.

- Do not say what you believe to be false; do not say that for which you lack adequate evidence.

- Be perspicuous, and specifically: avoid obscurity, avoid ambiguity, be brief; be orderly.

It does not take very much consideration to see that all the terms of Grice's maxims are highly culturally loaded. How much information is sufficient? What is relevant? What is clear and what is obscure? What counts as true?

English speakers are well-known to express their frustration with speakers of other languages who 'won't get to the point', who persist in spending time on 'irrelevant' matters, who are far from clear and who may not be adhering to the truth. To some extent there are more dangers of misunderstanding in CMC than in FV interactions. Problematic as crosscultural meetings may be, at least the interlocutors can see each other, look polite, make conciliatory gestures, rapidly adjust the drift of talk, correct a bad impression on the spot, and so on. All of these manæuvres are difficult if not impossible in CMC but may be partially overcome by multimedia developments.

The chief aspect in which cross-cultural misunderstandings are likely to persist is in relation to relevance. At the opening of a recent CMC master's course of mine where twenty-six English teachers were giving brief word-pictures of themselves, their interests and their work, the content that attracted most discussion was the word 'asshole' used by one of them, which some others considered taboo. A reasonable hypothesis is that language teachers would perceive a language issue as more relevant to their discussion than common interests like sport, which took a very secondary place. On another online course containing people from a range of subject areas, some (non-English) participants felt their contributions had been unfairly or even insultingly ignored. But it could be argued that what they had considered relevant was not perceived as such by the others. The phenomenon called 'flaming' - strong emotion expressed online in ways that would not be tolerated in FV - is likely to be at least partly due to such misunderstandings (Wang, 1996).

It may be pessimistic to assume that cultural differences and confusions are ineradicable, but at the very least, educators must be aware of, and make attempts to, safeguard against their worst effects. Again, careful framing of both topics and discussions is likely to be the best policy.

\section{Multimedia artefacts}

Print has been usurped by digital technology, so that it now incorporates multimedia and hypertext capabilities that have allowed the creation of new computer artefacts that have no generic name as yet, but which combine text, still and moving pictures, speech and musical sounds, in a fluid kaleidoscope of hyperlinked parts (Warschauer, 1999). In addition, the very fact that these artefacts are digitally stored, means that any user can both 'read' them in any order and alter them at will (Lanham, 1992). The combination of these features means we have something very unlike a book - a 'combook', where 'com' refers both to 'combination' and 'computer' (Pincas, 1998).

In fact the nature of literacy is altered. Words, a set of tokens that readers are compelled to interpret through their own thought processes, are now side by side with concrete 
realizations of meanings through icons and sounds. Iconic communication has been denigrated as 'comic book culture' (Lanham 1992). But there is another way of seeing it, namely as a new semiotic. Thus, icons, as much as words, need to be interpreted in the light of the context, the perceived goals of the originator, the cultural values within which these are set, and so forth. This is equally true of a moving image, of sounds and of music.

Icons of all kinds have the potential to function just like words, i.e. as mere tokens of whatever interpretations users wish to put upon them. They lead further and further into a new semiotic which will incorporate not only the old rhetorical traditions, but also visual and sound arts, and thus we are facing what Lanham calls a new organization of humanistic knowledge. It is beyond the scope of this paper to describe how knowledge is altered by the multimedia process, but that it is altered seems beyond doubt. My own faith is that it will enlarge our cognition, not confine it as Lanham suggests: 'Nothing less than human reason itself stands at risk' (Lanham 1992). More optimistic approaches are advocated by Kress and van Leeuwen (1996), Hill (1996), and Bolter (1991), among many.

None the less, the fact that combooks are digital and have hypertext introduces a fundamentally different process into literacy. The reader is no longer constrained by linear print to a 'fixed basic cognitive scale' and compelled to 'follow the argument on the level of generality the author has chosen to employ. With the new medium, the scale at which conceptual thought is pursued now becomes a user-selectable parameter' (Lanham 1992). In other words, the fact that the combook is on a computer not only permits the viewerreader to counterpoint sections in ways that the author may not have intended or foreseen, but further, to amend, rewrite, reformat, set in another typeface, change colours, all with a few keystrokes. Thus, the whole system of cultural authority enshrined in the book evaporates, literally, at a stroke. The fixity of print no longer operates and the 'reader' has turned into a potential author.

These two developments - multimedia and reader power - are most likely to have positive consequences for education, at least for a liberal cultural context. Access to combooks will become easier both cognitively and in cost, and they will be attractive to young learners because of the seamless interface with game software. Reasoning, judgement, logic, evaluation, will all be brought to bear on combooks. Whereas film or television, in their rigid form, possibly debased thought, a digital cluster of options can liberate it.

Nothing in this section should be taken to imply the demise of the traditional book. It is unlikely that the convenience of scanning and flipping pages of paper will be overtaken by even the smallest, most powerful, laptop. Although it is convenient for learners to have a whole study pack all together in one place, either in one book (or set of manuals), or on one CD-ROM disk or on a Web-page hyperlinked to other pages, it is well-known that students print out the contents of disks and Web pages. Perhaps we will see a 'combination combook', an integration of a paper book and a small laptop.

\section{The erosion of information boundaries}

In traditional writing it is a laborious process to cite other writers, even with established conventions. It is even more laborious, or indeed impossible, to cite conversations, lectures, television programmes, films, or any of the many aspects of our lives that we, personally, do not have a record of. But that will shortly change in a radical way. For instance, it is 
already possible for an email message to include a video clip. As distance education moves forward, and lectures are sent by video to students, and also as online discussions increase, all of these 'talked' texts will be available to be searched, copied, and cited.

Users are thereby liberated from the rigidity of print. Moreover, on the Internet, two further processes are occurring. These are the development of information retrieval facilities: (a) collecting items by keywords rather than by discipline boundaries, and (b) tracking citations within citations in published works, thus sweeping across information stores.

Because of both, conventional disciplines are melting into each other. The main difference between a keyword search and a citation search is that the former is dependent on knowledge of the field's vocabulary and on good formulation of the keyword(s), while the latter uses the expert authors themselves to lead to related literature through their own bibliographies and the further bibliographies contained in these, ad infinitum.

Although both the Social Sciences Citations Index and the Institute for Scientific Information index can be accessed via a Web browser, they are not yet able to scan the Web or read books. When they do, they will be able to add to their data of authored prose anything that is made available to the Web, such as sound and text conversations (synchronous chat and asynchronous email conferences) and all the elements of combooks.

Considerable adjustments will have to be made. We will soon have to give up our notions of being purveyors of prepackaged, finite, subjects-to-be-learned. And there are interacting processes here: as soon as users perceive the lack of boundaries, they behave differently. But that does not mean that education will become a free-for-all chaos. It does mean that we will have to tailor our courses so that they are seen as entry-points to wider exploration. Of course, 'good' education has always been somewhat like this, but it has become less possible for teachers and students alike to ignore the vast new potential.

\section{Conclusion}

Nowadays, the fear of added burdens is already well known among those who approach online education with a frightening vision of having to be ever at their computer screens. We can find gloomy pictures of 'an inevitable extension of working time and an intensification of work as faculty struggle at all hours of the day and night to stay on top of the technology and respond, via chat rooms, virtual office hours, and email, to both students and administrators to whom they have now become instantly and continuously accessible' (Noble, 1997).

Many teachers feel overwhelmed by the pressure to learn the new delivery modes - email, video-conferencing, the Internet. They fear not only that their own technical and academic competence may come into question, but that they will be deluged by constant availability to their students and a need to monitor information on the Internet in regular, exhausting ways.

This is not necessarily true. Taken together, the porosity of knowledge domains and the fluidity of combooks suggest that learners will collaborate not only with each other and their teachers, but within the very media they are exposed to. Transmission of knowledge is 
now tempered by a far higher degree of interaction between receiver and transmitter. Therefore the apparent burden on educators could be seen as decreased by the autonomous learning power of the individual and the increased opportunities for collaborative learning among students. This illuminates common remarks about the 'liberating' effect of the Internet.

A possible scenario, therefore, is that we shall see a regeneration of creativity as learners forge their own combooks, constructing a pastiche of conventional text with saved hyperlinked multimedia elements and citations retrieved from many divers sources. Ideas will, in principle at least, be preserved and reusable - truly recombinant knowledge.

\section{References}

Archee, R. (1993), 'Using computer mediated communication in an educational context: educational outcomes and pedagogical lessons of computer conferencing', Electronic Journal of Communication, 3 (2), http://www.cios.org/www/ejclv3n296.htm.

Bolter J. D. (1991), Writing Space: The Computer, Hypertext, and the History of Writing, Hillsdale, NJ: Lawrence Erlbaum Associates.

Condon, S. L. and Cech, C. G (1996), 'Discourse management strategies in face-to-face and computer-mediated decision making interactions', Electronic Journal of Communication, 6 (3), http://www.cios.org/wwwlejclv6n396.htm.

Coombes, N. (1993), 'The medium and the message', Electronic Journal of Communication, 3 (2), http://www.cios.org/www/ejclv3n296.htm.

Davis, B. H. and Brewer, J. P. (1997), Electronic Discourse, New York: SUNY Press.

Grice H. (1975), 'Logic and conversation', in P. Cole and J. Morgan (eds.), Syntax and Semantics Vol. 3, Speech Acts, New York: Academic Press, 41-58.

Hammonds, K. H., Jackson, S., George, G. D., Morris, K. (1997), 'The new university; a tough market is reshaping colleges', Business Week, 22,

http://www.businessweek.com/1997/51/b3558139.htm.

Harasim, L. M., Hiltz, S. R., Teles, L., Turoff, M. (1997), Learning Networks, A Field Guide to Teaching and Learning Online, Cambridge: MA: MIT Press.

Hershman, T. (1997), 'Speech recognition technology is becoming independent of speakers and languages', Byte, 10, http://www.byte.com/art/9710/sec17/art5.htm.

Hill, B. (1996), 'The metaphors of electronic communication', http://weber.u.washington.edul belindal.

Kachru, B. B. (1983), The Other Tongue, English across Cultures, London: Pergamon.

Kress G. and Leewen, T. van (1996), Reading Images: A Grammar of Visual Design, London: Routledge.

Lanham, R. A., (1992), The Implications of Electronic Information for the Sociology of Knowledge, http:/lenglish -server.hss. cmu.edulcyber/lanham.txt. 
Laurillard, D. (1993), Rethinking University Teaching: A Framework for the Effective Use of Educational Technology, London: Routledge.

Lewis, D. K. (1969), Convention: A Philosophical Study, Cambridge, MA: Harvard University Press.

Meyrowitz, J. (1985), No Sense of Space, London: Oxford University Press.

Noble, D., (1997), Digital Diploma Mills: The Automation of Higher Education on Email, owner-psyart\%LISTS.UFL.EDU@uk.ac.essex.mailhost (dated Mon. Dec. 15, 1997).

Pincas, A. (1999), 'Management issues for online courses ' Active Learning, 9, 41-5.

Pincas, A. (1999), 'Reference in Online Discourse', TESL-J online at <LISTSERV@CUNYVM.CUNY.EDU>.

Pincas, A. (1998), 'Porous knowledge domains, and new uses of language', Indian Journal of Open Learning, 7 (1), 129-42.

Poyatos, F. (1983), New Perspectives in Non-verbal Communication, London: Pergamon.

Shedletsky, L. J. (1993), 'Computer-mediated communication to facilitate seminar participation and active thinking: a case study', Electronic Journal of Communication, 3 (2), http://www.cios.org/www/ejclv3n296.htm.

Wang, H. (1996.), 'Flaming: more than a necessary evil for academic mailing lists', Electronic Journal of Communication, 6(1), http://www.cios.org/www/ejc/v6n196.htm.

Warschauer, M. (1999), Electronic Literacies, Hillsdale, NJ: Lawrence Erlbaum.

Yates, S. J. (1993), 'Speech, writing and computer conferencing, an analysis', in R. Mason (ed.), Computer Conferencing, The Last Word, Victoria, British Columbia, Canada: Beach Holme, 37-56.

Yates, S. J. (1995), 'Oral and written linguistic aspects of computer conferencing', in S. Herring (ed.), Computer Mediated Communication, Amsterdam, Netherlands: John Benjamins, 29-46. 